\title{
Clinical characteristics and outcomes of patients with severe acute respiratory infections (SARI): results from the Egyptian surveillance study 2010-2014
}

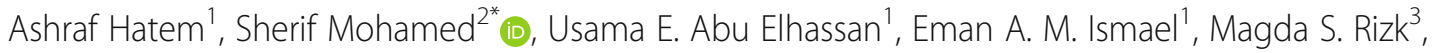
Amany El-kholy ${ }^{4}$ and Mohamed El-Harras ${ }^{5}$

\begin{abstract}
Background: Respiratory viral and atypical bacterial infections data in Egyptian patients are sparse. This study describes the clinical features and outcomes of patients with severe acute respiratory infections (SARI) in hospitalized patients in Egypt.
\end{abstract}

Methods: SARI surveillance was implemented at Cairo University Hospital (CUH) during the period 2010-2014. All hospitalized patients meeting the WHO case definition for SARI were enrolled. Nasopharyngeal/oropharyngeal (NP/ OP) swabs were collected and samples were tested using RT-PCR for influenza A, B, respiratory syncytial virus (RSV), human metapneumovirus (hMPV), parainfluenza virus (PIV 1,2,3,4), adenovirus, bocavirus, coronavirus, enterovirus, rhinovirus, and atypical bacteria. Data were analyzed to calculate positivity rates for viral pathogens and determine which pathogens related to severe outcomes or resulted in death.

Results: Overall, 1,075/3,207 (33.5\%) cases had a viral etiology, with a mean age of 5.74 ( \pm 13.87$)$ years. The highest rates were reported for RSV (485 cases, 45.2\%), PIV (125, 11.6\%), and adenovirus (105, 9.8\%). Children had a higher viral rate $(981,91.2 \%)$ compared to 94 (8.8\%) cases in adults. Patients with identified viruses had significantly lower rates for ICU admission, hospital stay, mechanical ventilation, and overall mortality than those without identified viruses. No infections were independently associated with severe outcomes.

Conclusions: Viral pathogens were encountered in one-third of hospitalized adult and pediatric Egyptian patients with SARI, while atypical bacteria had a minor role. Highest rates of viral infections were reported for RSV, PIV, and adenovirus. Viral infections had neither negative impacts on clinical features nor outcomes of patients with SARI in our locality.

Keywords: Clinical, Outcomes, Viral, SARI, Egypt, Surveillance

\section{Background}

The World Health Organization (WHO) estimates that acute respiratory infections (ARI) cause annual deaths approaching 4 million, at a rate of more than 60 deaths/ 100,000 populations [1]. Viruses are responsible for $30-70 \%$ of ARI where respiratory syncytial virus (RSV), influenza virus, parainfluenza virus (PIV), human Bocavirus, human metapneumovirus (hMPV), adenovirus,

\footnotetext{
* Correspondence: saawm220@gmail.com

${ }^{2}$ Department of Chest Diseases and Tuberculosis, Faculty of Medicine, Assiut University, Assiut 71516, Egypt

Full list of author information is available at the end of the article
}

rhinovirus, enterovirus and Coronaviruses account for the majority of these cases [1,2]. The 2009 influenza pandemic had highlighted the need for more global data on severe influenza disease, so the WHO recommended conducting surveillance for hospitalized severe acute respiratory infection (SARI), as well as influenza-like illness (ILI) in outpatients [3-6]. SARI surveillances are now conducted in many countries around the world; however, because of limited resources, they are only conducted in limited settings in the Middle East and Egypt [7-9]. Furthermore, the role of individual viral or

(c) The Author(s). 2019 Open Access This article is distributed under the terms of the Creative Commons Attribution 4.0 International License (http://creativecommons.org/licenses/by/4.0/), which permits unrestricted use, distribution, and 
atypical bacterial infection in causing ARI is not usually documented $[10,11]$.

In the current study, we analyzed surveillance data from Egyptian patients with SARI, enrolled at Cairo University Hospital (CUH) from 2010 to 2014. We aimed to calculate proportions of positive samples for different viral pathogens, to determine which pathogens were related to severe outcomes, and to address the impact of SARI on the clinical outcomes of enrolled patients, in terms of morbidity and mortality.

\section{Methods}

\section{Study population}

Cairo University Hospital (CUH) is a 5100-beds tertiary referral teaching hospital. Inclusion criteria consisted of hospitalized adults (defined as age $\geq 18$ years old), as well as pediatric patients (age $<18$ years old), with the diagnosis of SARI, who provided a respiratory sample, from February 2010 to February 2014. Due to an annual review by dedicated investigators and updates to WHO guidelines, the case definition for SARI has evolved over the study period. Before February 2010, as a global-surveillance case definition of SARI did not exist, the definition for SARI was adapted from the WHO protocol on rapid response for persons $\geq 5$ years old [3]. Whereas, for children $<5$ years old, SARI definition was adapted from the program for Integrated Management of Childhood Illness [4]. After March 2011, the global standards and tools for influenza surveillance developed by the WHO were adopted [5]. As of January 2014, the WHO surveillance case definitions for SARI was implemented [6] as follows, acute respiratory infection with history of fever or measured fever of $\geq$ $38 \mathrm{C}^{\circ}$; and cough; with onset within the last 10 days; and requiring hospitalization [6]. An enrollment form was used to collect data from enrolled eligible patients including patient demographics, medical history, clinical signs and symptoms, comorbidities, reported influenza vaccine status, recent travel history, treatment, clinical course, and outcome. Patients with incomplete medical records were excluded.

\section{Clinical samples and viral detection techniques}

Nasopharyngeal (NP) and oropharyngeal (OP) swabs for detecting viruses and blood cultures for detecting bacteria were taken from eligible patients on admission using operating procedures described by the WHO [12]. Specimens were taken an average of 7 days after illness onset (range: 1-66 days).

Total nucleic acid (TNA) was extracted by the automated KingFisher Flex Magnetic Particle Processor (Thermo Scientific, Waltham, MA, USA) using MagMAX Total Nucleic Acid Isolation Kit (Cat No. AM 1840, Applied Biosystems, Foster, CA, USA) according to the manufacturer's instructions. The viral target was amplified using specific primers and probes produced by the CDC (Atlanta, GA, USA) and following standard protocol for reverse transcription polymerase chain reaction detection. From 2010 to 2012, testing for RSV, adenovirus, human parainfluenza viruses (hPIV) 1, 2 and 3, influenza (A and B) and human metapneumovirus was conducted at $\mathrm{CUH}$ laboratory and sent for confirmation by the Naval Medical Research Unit No.3 (NAMRU-3) laboratory. From 2013 to 2014, testing was conducted at CUH laboratory. For all samples, the human RNase P gene (RP) was tested as an internal positive control to ensure proper sample collection and nucleic acid extraction. Samples were considered positive to the viral target if the amplification curve crossed the threshold line before cycle 40. All clinical samples should be positive to RP with cutoff value $\leq 37$, as prescribed previously [8]. Blood samples were collected for detection of Mycoplasma pneumonia, Chlamydia pneumonia, and Legionella pneumophila, using RT-qPCR.

\section{Ethical standards}

Prior to study initiation, the study protocol was reviewed and approved by Institutional Review Board at the NAMRU-3, as well as the ethical committee of CUH, in compliance with all applicable federal U.S. regulations governing the protection of human subjects. An informed written consent was obtained from the patients (in the case of adult patients) or patients' parent/legal guardian (in the case of pediatric patients).

\section{Statistical analysis}

Data analyses were conducted using the software SPSS (Statistical Package for the Social Science; IBM Corp, NY, USA); version 22. Data were summarized using median (range) for quantitative variables and number and percent for qualitative variables. Comparison between groups was done using the Chi-square test for qualitative variables, independent sample t-test for normally distributed quantitative variables, while the Mann-Whitney $U$ test was used for quantitative variables that are not normally distributed. Indicators of severe disease were assessed for each pathogen of interest using Mantel-Haenszel estimates to calculate odds ratios and confidence intervals and the MantelHaenszel chi-squared test to assess statistical significance [13]. Logistic regression was used to examine associations between viral respiratory pathogens and severe outcomes, defined as illness requiring ventilation or intensive care unit (ICU) or resulting in death while controlling for demographic and clinical characteristics. Only variables with statistically significant univariate association with severe outcomes were included in multivariate regression analysis. All tests were two-sided, and differences with $p<0.05$ were considered significant. 


\section{Results}

\section{Demographic data of the study population}

Out of 3,207 participants enrolled in this SARI surveillance, 1,075 (33.5\%) had positive results for viral and atypical bacteria tested. They included 569/1,075 (53\%) females and 506/1,075 (47\%) males. The median age was one year (range 0-85 years). Children less than 18 years had a higher viral etiology (981 patients, 91.2\%) compared to $94(8.8 \%)$ ones in adults. Notably, children $<5$ years represented $83 \%$ of patients. The highest rates of viral infections were reported for RSV (485 patients, $45.2 \%)$, PIV (125, 11.6\%), and adenovirus (105, 9.8\%). Other encountered viruses included rhinovirus, hMPV, and BOCA virus $(2 \%, 7 \%$, and $1 \%$, respectively). Only 3 cases were positive for Mycoplasma and were co-infected with RSV, while only one case of Chlamydia was co-infected with RSV and hMPV. Neither Coronavirus nor Legionella was detected. Table 1 shows these data.

\section{Clinical characteristics of viral-infected versus no virus- detected individuals}

In comparison to non-viral infected individuals, viral-infected SARI ones had significantly predominant signs and symptoms at presentation. Particularly, they had significant viral prodromal symptoms, as well as tachypnea, wheezes, and convulsions ( $\mathrm{p}=0.000$ each). Among individual viral pathogens, SARI patients with influenza had more significant tachypnea $(p=0.038)$, wheezes $(p=0.000)$, and abnormal breath sounds $(p=$ 0.023 ), than those with non-influenza viral infections. Patients whose specimens were collected within 5 days of the onset of symptoms were more likely to have a viral pathogen detected than those whose specimens were collected later ( $73 \%$ versus $36 \%, \mathrm{p}=0.047$ ).

Fifty-three percent of patients had at least one underlying medical condition. These comorbidities included chronic respiratory disorders (asthma, COPD, bronchiectasis, and immotile cilia syndrome), cardiac disorders (heart failure congenital heart diseases, and cardiomyopathy), neuromuscular disorders (epilepsy, cerebral palsy, and myopathies), hematological disorders (thalassemia), endocrine disorders (diabetes mellitus, hypothyroidism, and morbid obesity), renal disorders (end-stage renal disease), and liver disorders (liver cirrhosis and hepatic failure).

Patients with comorbidities $(n=570,53 \%)$ were significantly older compared to those with no comorbidities (median age: 54 versus $3, p<0.001$ ). Additionally, they were significantly more likely to be symptomatic.

In terms of comorbidities, patients with and without viral detection differed significantly in the frequencies of chronic respiratory $(\mathrm{p}=0.002)$, endocrine $(\mathrm{p}=0.000)$, hepatic $(\mathrm{p}=0.002)$, and neuromuscular disorders $(\mathrm{p}=0.001)$. Among individual viral pathogens, SARI patients with para-influenza virus had significant endocrine $(p=0.004)$, and neuromuscular disorders $(\mathrm{p}=0.012)$, than those with non-para-influenza viral infections.

For influenza vaccination history; 832/1,075 (77.4\%) cases did not receive the vaccine within the 12 months prior to hospital admission, while 243/1,075 (22.6\%) were reported as unknown for an influenza vaccination status. Table 1 details these results.

\section{Clinical course, complications, and outcomes in viral- infected patients}

In comparison to non-viral infected individuals, viral-infected SARI ones had significantly lower rates of pneumonia $(p=0.004)$ and admission to the ICU $(\mathrm{p}=0.000)$. Patients with influenza virus tended to have significantly different rates of admission to the ICU $(\mathrm{p}=0.045)$, and mechanical ventilation $(\mathrm{p}=0.001)$, in comparison to those with non-influenza infections. With regards to complications, viral-infected SARI patients had significant differences for developing respiratory failure $(\mathrm{p}=0.033)$, and acute respiratory distress syndrome; ARDS ( $\mathrm{p}=0.011)$, in comparison to those without viral infections.

Overall mortality in SARI-positive patients was 24/ $1,075(2.2 \%)$ and peaked at $1 \%$ in 2014 . Overall, only $2(8 \%)$ were adults, while $22(92 \%)$ were children. Among children, $18(75 \%)$ were aged $<5$ years. Overall, two-thirds $(16 / 24)$ had comorbidities. All patients who died were admitted to the ICU and mechanically ventilated. Notably, all patients who died tested positive for a viral pathogen; twelve were positive for RSV, four for influenza virus, two for adenovirus, one for hMPV, one for PIV and four for mixed viral infections, respectively. Among those who died, there was a significant difference between those with (2.2\%) and without (5\%) viral detection $(p=0.005)$. Among individual viral pathogens, SARI patients with RSV and influenza had significant deaths $(p=0.045$ and 0.006$)$, in comparison to those with non-RSV and non-influenza viral infections. No mortality was reported for patients with atypical bacteria (Table 1).

\section{Severe outcomes in viral-infected patients}

No infections were independently associated with increased severity of SARI, as indicated by illness requiring mechanical ventilation and/or ICU and/or resulting in death. There was strong evidence that individuals with RSV and influenza were less likely to experience a severe outcome than those not infected with each of these pathogens (RSV OR 1.433, 95\% CI 4.698-6.132. $\mathrm{p}=0.021$, influenza OR 3.937, 95\% CI 2.447-6.3340, $\mathrm{p}=0.000$ ). Individuals with multiple infections were no more likely than those with infection with a single pathogen to experience severe outcomes (OR 0.232, 95\% CI 0.155-0.619, $\mathrm{p}=0.240$ ). 
Hater et al. Multidisciplinary Respiratory Medicine $\quad$ (2019) 14:11

Page 4 of 12

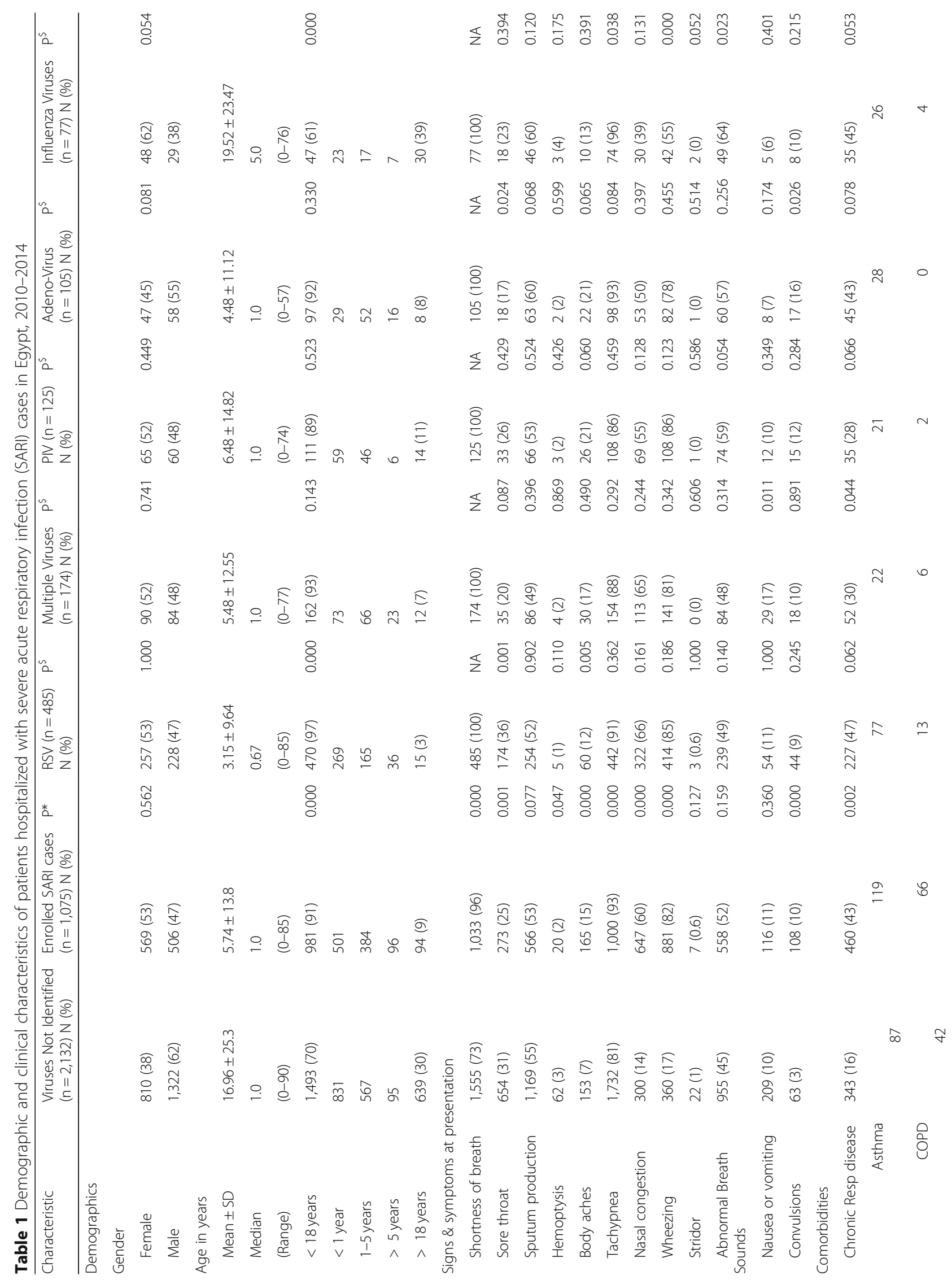


Hater et al. Multidisciplinary Respiratory Medicine

(2019) 14:11

Page 5 of 12

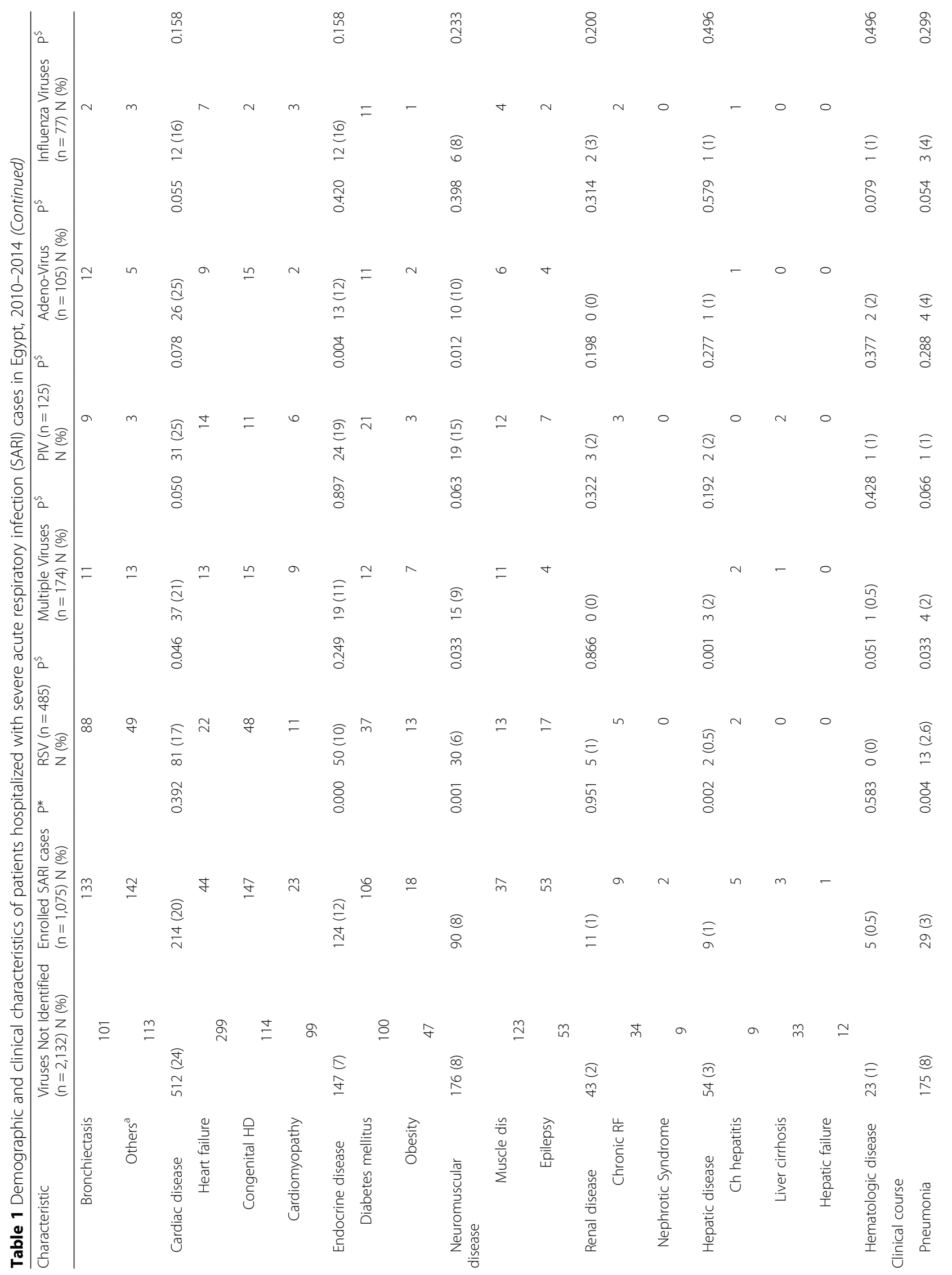




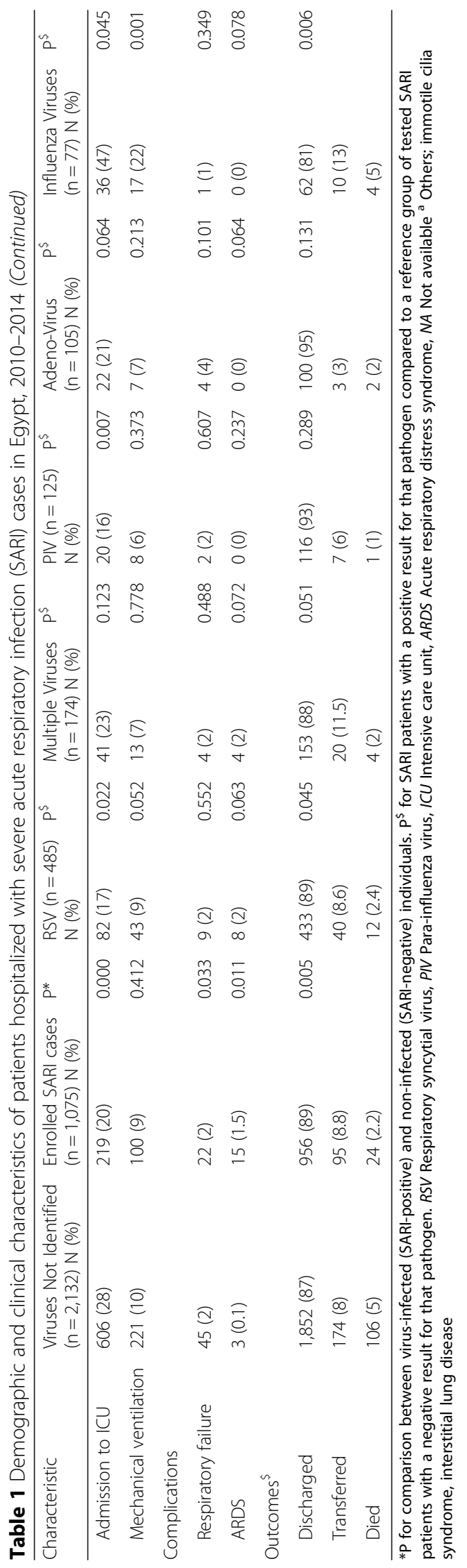


When analyses were stratified by age, neither significant differences in severe outcomes could be encountered between viral-infected and non-infected individuals (OR 0.983, 95\% CI 0.503-1.924, $\mathrm{p}=0.961$ and OR 1.100, 95\% CI 0.704-1.718, p=0.675) nor between individual viral infections, among children and adults. Table 2 shows these details (Data for PIV, hMPV, Boca virus, rhino-, and enterovirus are not shown in the table).

Logistic regression was used to further examine associations with severe outcomes in SARI-positive individuals with complete demographic data and clinical risk factors. By univariate analysis, individuals with positive results for rhinovirus and adults $>18$ years were more likely to experience a severe outcome than those not infected with rhinovirus (OR 4.975, 95\% CI 2.431-17.812, $\mathrm{p}=0.024)$ and children $<18$ years (OR 10.357, 95\% CI 5.895-18.197, $\mathrm{p}=0.000$ ), respectively.

Multivariate analysis confirmed these results where individuals with positive results for rhinovirus and adults $>18$ years were more likely to experience a severe

Table 2 Indicators of the severity of SARI by pathogen and age

\begin{tabular}{|c|c|c|c|c|c|c|c|c|c|c|c|c|c|c|c|}
\hline & \multicolumn{3}{|c|}{ SARI cases } & \multicolumn{3}{|l|}{ RSV } & \multicolumn{3}{|c|}{ Adenovirus } & \multicolumn{3}{|c|}{ Influenza } & \multicolumn{3}{|c|}{ Multiple Viruses } \\
\hline & No $(\%)$ & $\begin{array}{l}\text { OR }(95 \% \\
\text { CI) }\end{array}$ & $P^{*}$ & No (\%) & $\begin{array}{l}\text { OR }(95 \% \\
\mathrm{Cl})\end{array}$ & $P^{\$}$ & No $(\%)$ & $\begin{array}{l}\text { OR }(95 \% \\
\mathrm{CI})\end{array}$ & $P^{\$}$ & No (\%) & $\begin{array}{l}\text { OR }(95 \% \\
\mathrm{Cl})\end{array}$ & $P^{\$}$ & No $(\%)$ & $\begin{array}{l}\text { OR }(95 \% \\
\mathrm{Cl})\end{array}$ & $P^{\$}$ \\
\hline \multicolumn{16}{|l|}{ All participants } \\
\hline Ventilation & $\begin{array}{l}100 / \\
1,075 \\
(9)\end{array}$ & $\begin{array}{l}1.280 \\
(0.703- \\
2.329\end{array}$ & 0.419 & $\begin{array}{l}43 / \\
485 \\
(9)\end{array}$ & $\begin{array}{l}0.177 \\
(-1.005- \\
0.185)\end{array}$ & 0.866 & $\begin{array}{l}7 / 105 \\
(7)\end{array}$ & $\begin{array}{l}1.293 \\
(0.682- \\
2.452)\end{array}$ & 0.431 & $\begin{array}{l}17 / 77 \\
(22)\end{array}$ & $\begin{array}{l}3.123 \\
(1.743- \\
5.598)\end{array}$ & 0.000 & $\begin{array}{l}13 / \\
174 \\
(7)\end{array}$ & $\begin{array}{l}0.755 \\
\left(0.412^{-}\right. \\
1.386)\end{array}$ & 0.365 \\
\hline $\mathrm{ICU}$ & $\begin{array}{l}219 / \\
1,075 \\
(20)\end{array}$ & $\begin{array}{l}0.972 \\
(0.706- \\
1.337)\end{array}$ & 0.861 & $\begin{array}{l}82 / \\
485 \\
(17)\end{array}$ & $\begin{array}{l}1.897 \\
(12.591- \\
13.635)\end{array}$ & 0.017 & $\begin{array}{l}22 / \\
105 \\
(21)\end{array}$ & $\begin{array}{l}1.040 \\
(0.634- \\
1.707)\end{array}$ & 0.876 & $\begin{array}{l}36 / 77 \\
(47)\end{array}$ & $\begin{array}{l}3.910 \\
(2.431- \\
6.290)\end{array}$ & 0.000 & $\begin{array}{l}41 / \\
174 \\
(23)\end{array}$ & $\begin{array}{l}0.225 \\
(0.162- \\
0.612)\end{array}$ & 0.254 \\
\hline Death & $\begin{array}{l}24 / \\
1,075 \\
(2)\end{array}$ & $\begin{array}{l}0.00 \\
(0.941- \\
0.966)\end{array}$ & 0.557 & $\begin{array}{l}12 / \\
485 \\
(2)\end{array}$ & $\begin{array}{l}0.530 \\
(-1.215- \\
0.625)\end{array}$ & 0.637 & $\begin{array}{l}2 / 105 \\
(2)\end{array}$ & $\begin{array}{l}0.837 \\
(0.194- \\
3.609)\end{array}$ & 0.811 & $\begin{array}{l}4 / 77 \\
(5)\end{array}$ & $\begin{array}{l}0.986 \\
(-0.114- \\
2.085)\end{array}$ & 0.079 & $\begin{array}{l}4 / 174 \\
(2)\end{array}$ & $\begin{array}{l}0.036 \\
(1.050- \\
1.122)\end{array}$ & 0.948 \\
\hline $\begin{array}{l}\text { Severe } \\
\text { Outcome }\end{array}$ & $\begin{array}{l}219 / \\
1,075 \\
(20)\end{array}$ & $\begin{array}{l}0.972 \\
(0.706- \\
1.337)\end{array}$ & 0.861 & $\begin{array}{l}82 / \\
485 \\
(17)\end{array}$ & $\begin{array}{l}1.433 \\
\left(4.698^{-}\right. \\
6.132)\end{array}$ & 0.021 & $\begin{array}{l}22 / \\
105 \\
(21)\end{array}$ & $\begin{array}{l}1.047 \\
(0.638- \\
1.738)\end{array}$ & 0.857 & $\begin{array}{l}36 / 77 \\
(47)\end{array}$ & $\begin{array}{l}3.937 \\
(2.447- \\
6.334)\end{array}$ & 0.000 & $\begin{array}{l}41 / \\
174 \\
(23)\end{array}$ & $\begin{array}{l}0.232 \\
(0.155- \\
0.619)\end{array}$ & 0.240 \\
\hline \multicolumn{16}{|c|}{ Children < 18 years } \\
\hline Ventilation & $\begin{array}{l}66 / \\
981 \\
(7)\end{array}$ & $\begin{array}{l}1.032 \\
(0.305- \\
3.541)\end{array}$ & 0.952 & $\begin{array}{l}36 / \\
485 \\
(8)\end{array}$ & $\begin{array}{l}0.894 \\
(0.566- \\
1.413)\end{array}$ & 0.632 & $\begin{array}{l}7 / 105 \\
(7)\end{array}$ & $\begin{array}{l}0.907 \\
(04.05- \\
2.032)\end{array}$ & 0.812 & $\begin{array}{l}5 / 77 \\
(6)\end{array}$ & $\begin{array}{l}1.785 \\
(0.676- \\
4.713)\end{array}$ & .0243 & $\begin{array}{l}13 / \\
174 \\
(7)\end{array}$ & $\begin{array}{l}0.995 \\
(0.536- \\
1.849)\end{array}$ & 0.988 \\
\hline ICU & $\begin{array}{l}178 / \\
981 \\
(18)\end{array}$ & $\begin{array}{l}0.983 \\
(0.503- \\
1.924)\end{array}$ & 0.961 & $\begin{array}{l}68 / \\
485 \\
(14)\end{array}$ & $\begin{array}{l}0.838 \\
(0.594- \\
1.182)\end{array}$ & 0.314 & $\begin{array}{l}11 / \\
105 \\
(10)\end{array}$ & $\begin{array}{l}0.691 \\
(0359- \\
1.330)\end{array}$ & 0.268 & $\begin{array}{l}10 / 77 \\
(13)\end{array}$ & $\begin{array}{l}2.008 \\
(0.952- \\
(4.237)\end{array}$ & 0.067 & $\begin{array}{l}32 / \\
174 \\
(18)\end{array}$ & $\begin{array}{l}1.418 \\
(0.921- \\
2.185)\end{array}$ & 0.113 \\
\hline Death & $\begin{array}{l}13 / \\
981 \\
(1)\end{array}$ & $\begin{array}{l}0.973 \\
(0.960- \\
(0.987)\end{array}$ & 0.784 & $\begin{array}{l}12 / \\
485 \\
(2)\end{array}$ & $\begin{array}{l}1.363 \\
(0.583- \\
1.385)\end{array}$ & 0.474 & $\begin{array}{l}2 / 105 \\
(2)\end{array}$ & $\begin{array}{l}0.966 \\
(0.222- \\
4.198)\end{array}$ & 0.963 & $\begin{array}{l}2 / 77 \\
(2)\end{array}$ & $\begin{array}{l}2.640 \\
(0.594- \\
11.740)\end{array}$ & 0.202 & $\begin{array}{l}4 / 174 \\
(2)\end{array}$ & $\begin{array}{l}1.162 \\
\left(0.338^{-}\right. \\
3.479)\end{array}$ & 0.789 \\
\hline $\begin{array}{l}\text { Severe } \\
\text { Outcome }\end{array}$ & $\begin{array}{l}178 / \\
981 \\
(18)\end{array}$ & $\begin{array}{l}0.983 \\
(0.503- \\
1.924)\end{array}$ & 0.961 & $\begin{array}{l}68 / \\
485 \\
(14)\end{array}$ & $\begin{array}{l}0.849 \\
(0.602- \\
1.199)\end{array}$ & 0.353 & $\begin{array}{l}11 / \\
105 \\
(10)\end{array}$ & $\begin{array}{l}0.697 \\
(0.362- \\
1.342)\end{array}$ & 0279 & $\begin{array}{l}10 / 77 \\
(13)\end{array}$ & $\begin{array}{l}2.024 \\
(0.959- \\
4.271)\end{array}$ & 0.064 & $\begin{array}{l}32 / \\
174 \\
(18)\end{array}$ & $\begin{array}{l}1.432 \\
(0929- \\
2.206)\end{array}$ & 0.114 \\
\hline \multicolumn{16}{|c|}{ Adults $>18$ years } \\
\hline Ventilation & $\begin{array}{l}34 / 94 \\
(36)\end{array}$ & $\begin{array}{l}1.357 \\
(0.645- \\
2.856)\end{array}$ & 0.421 & $\begin{array}{l}7 / 485 \\
(1)\end{array}$ & $\begin{array}{l}0.966 \\
(0.114- \\
2.085)\end{array}$ & 0.078 & $\begin{array}{l}0 / 105 \\
(0)\end{array}$ & $\begin{array}{l}2.991 \\
(0.851- \\
10.514)\end{array}$ & 0.088 & $\begin{array}{l}12 / 77 \\
(16)\end{array}$ & $\begin{array}{l}2.878 \\
(1014- \\
8.166)\end{array}$ & 0.052 & $\begin{array}{l}0 / 174 \\
(0)\end{array}$ & $\begin{array}{l}0.787 \\
(0.669- \\
0.885)\end{array}$ & 0.063 \\
\hline $\mathrm{ICU}$ & $\begin{array}{l}41 / 94 \\
(44)\end{array}$ & $\begin{array}{l}1.100 \\
(0.704- \\
1.718)\end{array}$ & 0.675 & $\begin{array}{l}14 / \\
485 \\
(3)\end{array}$ & $\begin{array}{l}1.493 \\
(0.282- \\
2.452)\end{array}$ & 0.527 & $\begin{array}{l}11 / \\
105 \\
(10)\end{array}$ & $\begin{array}{l}3.235 \\
(0.671- \\
15.593)\end{array}$ & 0.143 & $\begin{array}{l}26 / 77 \\
(34)\end{array}$ & $\begin{array}{l}0.929 \\
(0.392- \\
2.198)\end{array}$ & 0.866 & $\begin{array}{l}9 / 174 \\
(5)\end{array}$ & $\begin{array}{l}0.606 \\
(0.203- \\
1.815)\end{array}$ & 0.371 \\
\hline Death & $\begin{array}{l}11 / 94 \\
(12)\end{array}$ & $\begin{array}{l}0.934 \\
\left(0.912^{-}\right. \\
0.965)\end{array}$ & 0.793 & $\begin{array}{l}0 / 485 \\
(0)\end{array}$ & $\begin{array}{l}0.848 \\
(0.437- \\
1.196)\end{array}$ & 0.691 & $\begin{array}{l}11 / \\
105 \\
(10)\end{array}$ & $\begin{array}{l}0.859 \\
\left(0.790^{-}\right. \\
0.933)\end{array}$ & 0.645 & $\begin{array}{l}2 / 77 \\
(2)\end{array}$ & $\begin{array}{l}0.413 \\
(0.324- \\
0.527)\end{array}$ & 0.351 & $0(0)$ & $\begin{array}{l}0.826 \\
(0.752- \\
0.907)\end{array}$ & 0.763 \\
\hline $\begin{array}{l}\text { Severe } \\
\text { Outcome }\end{array}$ & $\begin{array}{l}41 / 94 \\
(44)\end{array}$ & $\begin{array}{l}1.100 \\
(0.704- \\
1.718)\end{array}$ & 0.675 & $\begin{array}{l}14 / \\
485 \\
(3)\end{array}$ & $\begin{array}{l}1.493 \\
(0.282- \\
2.452)\end{array}$ & 0.527 & $\begin{array}{l}0 / 105 \\
(0)\end{array}$ & $\begin{array}{l}3.235 \\
(0.671- \\
15.593)\end{array}$ & 0.143 & $\begin{array}{l}26 / 77 \\
(34)\end{array}$ & $\begin{array}{l}0.929 \\
(0.392- \\
2.198)\end{array}$ & 0.866 & $\begin{array}{l}9 / 174 \\
(5)\end{array}$ & $\begin{array}{l}0.606 \\
(0.203- \\
1.815)\end{array}$ & 0.371 \\
\hline
\end{tabular}

*P for comparison between virus-infected (SARI-positive) and non-infected (SARI-negative) individuals. $P^{\$}$ for SARI patients with a positive result for that pathogen compared to a reference group of tested SARI patients with a negative result for that pathogen. RSV Respiratory syncytial virus, PIV Para-influenza virus, ICU Intensive care unit, Severe outcome is defined as illness requiring ventilation or ICU or resulting in death 
outcome than those not infected with rhinovirus (OR 4.807, 95\% CI 2.981-16.112, $\mathrm{p}=0.025)$ and children $<18$ years (OR 11.716, 95\% CI 7.225-18.998, $\mathrm{p}=0.000$ ), respectively.

Table 3 shows these results.

\section{Comparison between RSV-positive and other viral cases}

Being the most commonly detected virus among our cohort, clinical characteristics and outcomes of RSV-positive

Table 3 Univariate and multivariate logistic regression for predictors of severe outcomes among viral-infected SARI cases

\begin{tabular}{|c|c|c|c|}
\hline & Univariate A & nalysis & \\
\hline & & OR $(95 \% \mathrm{Cl})$ & $P$ \\
\hline RSV & Negative & ref & \\
\hline & Positive & $0.00(-)$ & 0.989 \\
\hline Adenovirus & Negative & ref & \\
\hline & Positive & $0.927(0.533-1.612)$ & 0.788 \\
\hline Rhinovirus & Negative & ref & \\
\hline & Positive & $4.975(2.431-17.812)$ & 0.024 \\
\hline Enterovirus & Negative & ref & \\
\hline & Positive & $0.00(-)$ & 1.000 \\
\hline Influenza & Negative & ref & \\
\hline & Positive & $1.150(0.608-2.176)$ & 0.667 \\
\hline BOCA virus & Negative & ref & \\
\hline & Positive & $0.413(0.051-3.371)$ & 0.409 \\
\hline HMPV & Negative & ref & \\
\hline & Positive & $0.845(0.431-1.656)$ & 0.624 \\
\hline PIV & Negative & ref & \\
\hline & Positive & $0.633(0.361-1.112)$ & 0.112 \\
\hline Multiple viruses & Single virus & ref & \\
\hline & Multiple viruses & $1.515(0.974-2.357)$ & 0.065 \\
\hline Age & Adults $>18$ years & ref & \\
\hline & Children <18 years & $10.357(5.895-18.197)$ & 0.000 \\
\hline Gender & Male & ref & \\
\hline & Female & $0.893(0.643-1.239)$ & 0.497 \\
\hline Comorbidities & None & ref & \\
\hline & Any & $1.181(0.840-1.661)$ & 0.338 \\
\hline Multivariate anal & & & \\
\hline & & OR $(95 \% \mathrm{Cl})$ & $P$ value \\
\hline Rhinovirus & Negative & ref & \\
\hline & Positive & $4.807(2.981-16.112)$ & 0.025 \\
\hline Age & Adults $>18$ years & ref & \\
\hline & Children $<18$ years & 11.716 (7.225-18.998) & 0.000 \\
\hline
\end{tabular}

RSV Respiratory syncytial virus, hMPV Human metapneumovirus, PIV Parainfluenza virus, OR Odds ratio patients were compared to those with other respiratory positive cases as well as viral-negative patients.

While patients with RSV-positive infections had significant differences with those with no respiratory viruses identified, with regards to clinical signs and symptoms, comorbidities, and outcomes (ICU admission and deaths); they had no differences with those tested positive for other viral pathogens, with regards to the same parameters. (Table 4 shows these details)

\section{Severe outcomes in RSV-positive patients}

Logistic regression was used to examine associations with severe outcomes in RSV-positive patients with complete demographic data and clinical risk factors. By univariate analysis, individuals with RSV and associated comorbidities were more likely to experience severe outcomes (OR 4.703, 95\% CI 0.803-9.672, $\mathrm{p}=0.001$ ) than those with RSV and no comorbidities (Table 5).

\section{Discussion}

To the best of our knowledge, this is the largest surveillance Egyptian study that addressed the epidemiological patterns of SARI due to viruses and atypical bacteria in both children and adult population and their relation to the clinical characteristics and outcomes of those patients.

The worldwide distribution of viral etiology as a cause of SARI varies between $2 \%$ and up to $78 \%[7,11,13,14]$. In this study, we found a viral etiology in $33.5 \%$ of hospitalized patients with SARI, which is comparable to previous studies conducted in either developing or Middle Eastern countries [9-11, 13]. The finding that two-thirds of SARI cases had no pathogen detected suggests that poor or late specimen collection may have contributed to a lower yield of detected viruses. Interestingly, children $<18$ years represented the majority (91.2\%) of our cohort. Notably, this contradicts findings observed by other studies $[14,15]$. In their surveillance for SARI in Northern Vietnam, Nguyen et al [15] observed that $22.7 \%$ of their cohort were children $<18$ years, while $77.3 \%$ were adults $>18$ years. Again, children $<5$ years represented $83 \%$ of our cases. This is in accordance with those surveillance data from Southern Arizona, 82\% [14], lower than those from China (94\% in $<72$ months) [16] and higher than in Kenya,71\% [11].

The highest rates of viral infections were reported for RSV (45.2\%), PIV (11.6\%), and adenovirus (9.8\%), with a relatively low rate $(7.2 \%)$ for influenza viruses. Not unexpected, RSV was the most predominant respiratory virus with a prevalence of $45 \%$; emphasizing its role as the major cause of SARI in infants and young children worldwide [7, $8,13-17]$. Notably, the proportion of SARI cases positive for RSV in children $<5$ years in our surveillance $(90 \%)$ was markedly higher than those reported in surveillance data 
Table 4 Comparison of SARI patients with RSV to those with a non-RSV or to those with no respiratory virus identified

\begin{tabular}{|c|c|c|c|c|c|}
\hline Characteristic & RSV-positive $(n=485) N(\%)$ & Other Viruses Positive $(n=590) N(\%)$ & $P^{*}$ & $\begin{array}{l}\text { No virus Identified } \\
(n=2132) N(\%)\end{array}$ & $p^{\$}$ \\
\hline \multicolumn{6}{|l|}{ Gender } \\
\hline Female & $257(53)$ & $312(53)$ & 0.203 & $810(38)$ & 0.000 \\
\hline Male & $228(47)$ & $278(47)$ & & $1,322(62)$ & \\
\hline \multicolumn{6}{|l|}{ Age } \\
\hline Below 18 y & $470(97)$ & $511(87)$ & 0.801 & $1,493(70)$ & 0.373 \\
\hline Above 18 y & $15(3)$ & $79(13)$ & & $639(30)$ & \\
\hline Symptom onset $\leq 7$ days & $456(94)$ & $480(81)$ & 0.064 & $1,211(57)$ & 0.250 \\
\hline Cough & $485(100)$ & $536(91)$ & 0.882 & $2,132(100)$ & 1.000 \\
\hline SOB & $485(100)$ & $548(93)$ & 1.000 & $1,555(73)$ & 0.077 \\
\hline Fever & $485(100)$ & $590(100)$ & 1.000 & $2,132(100)$ & 1.000 \\
\hline Sore throat & $174(36)$ & $311(53)$ & 0.000 & $654(31)$ & 0.000 \\
\hline Sputum production & $254(52)$ & $312(53)$ & 1.000 & $1,169(55)$ & 0.870 \\
\hline Body aches & $60(12)$ & $105(18)$ & 0.063 & $153(7)$ & 0.004 \\
\hline Tachypnea & $442(91)$ & $558(95)$ & 0.063 & $1,732(81)$ & 0.087 \\
\hline Nasal congestion & $322(66)$ & $325(55)$ & 1.000 & $300(14)$ & 0.060 \\
\hline Wheezing & $414(85)$ & $467(79)$ & 0.031 & $360(17)$ & 0.063 \\
\hline Abnormal BS & $239(49)$ & $319(54)$ & 0.008 & $955(45)$ & 0.022 \\
\hline Nausea or vomiting & $54(11)$ & $62(10)$ & 0.988 & $209(10)$ & 0.003 \\
\hline Convulsions & $44(10)$ & $64(11)$ & 0.677 & $63(3)$ & 0.046 \\
\hline pneumonia & $13(3)$ & $16(3)$ & 1.000 & $175(8)$ & 0.001 \\
\hline Chronic lung disease & $227(47)$ & $233(39)$ & 0.086 & $343(16)$ & 0.001 \\
\hline Cardiac disease & $81(17)$ & $133(23)$ & 0.063 & $512(24)$ & 0.022 \\
\hline Endocrine disease & $50(10)$ & $74(13)$ & 0.866 & $147(7)$ & 0.002 \\
\hline Renal disease & $5(1)$ & $6(1)$ & 1.000 & $43(2)$ & 0.246 \\
\hline Neuromuscular disorder & $30(6)$ & $60(10)$ & 0.333 & $176(8)$ & 0.033 \\
\hline ICU & $82(17)$ & $137(23)$ & 0.121 & $606(28)$ & 0.000 \\
\hline Ventilation & $43(9)$ & $57(10)$ & 0.473 & $221(10)$ & 0.343 \\
\hline ARDS & $8(1)$ & $7(1)$ & 1.000 & $3(0)$ & 0.000 \\
\hline Respiratory Failure & $9(2)$ & $13(2)$ & 0.988 & $45(2)$ & 1.000 \\
\hline Death & $12(2)$ & $12(2)$ & 1.000 & $106(5)$ & 0.000 \\
\hline
\end{tabular}

*P for comparison for SARI patients with a positive result for RSV (RSV-positive SARI patients) and a reference group of tested SARI patients with a negative result for RSV (non-RSV-positive SARI patients).P\$ for comparison between RSV-positive SARI patients and non-infected individuals (SARI-negative individuals); RSV Respiratory syncytial virus, SOB Shortness of breath, ICU Intensive care unit, ARDS Acute respiratory distress syndrome

Table $\mathbf{5}$ Logistic regression for predictors of severe outcomes for RSV-positive cases

\begin{tabular}{llll}
\hline Univariate analysis & & \\
\hline \multirow{2}{*}{ Gender } & Male & OR (95\% Cl) & P \\
& Female & ref & \\
Age & Adults >18 years & ref & \\
& Children <18 years & $1.600(0.400-6.163)$ & 0.086 \\
Comorbidities & None & ref & \\
& Any & 4.703(0.803-9.672) & 0.001
\end{tabular}

RSV Repiratory syncytial virus, OR Odds ratio from Kenya, 21\% [11], Southern Arizona, 31\% [14], and even higher than previous studies in Egypt [18].

We observed that, SARI cases $<5$ years were significantly more likely than older patients to be infected with each of the pathogens examined, particularly for RSV and influenza. As the majority of enrolled patients were children (83\%), this is not unexpected since these pathogens have a strong association with this age group. This is inconsistent with data that nearly $80 \%$ of children are exposed to RSV by age two, $100 \%$ to hMPV by age five and $90 \%$ to hPIV by age five [19]. Furthermore, hPIV is a significant etiology of LRTI in children [20], second only to RSV [21], and adenoviruses are the second most 
common viral pathogen in children under two years of age [7].

Notably, our results showed a very minor role for atypical bacteria in causing SARI in our locality. Only 3 cases were positive for Mycoplasma (co-infected with RSV), while only one case of Chlamydia was co-infected with RSV and hMPV. Clinical presentations differed significantly between those with non-viral infected individuals and viral-infected SARI ones. The later had significant viral prodromal symptoms, as well as tachypnea, wheezes, and convulsions. Furthermore, SARI patients with influenza had significant tachypnea, wheezes, and abnormal breath sounds, than those with non-influenza viral infections. The presence of these signs at presentation could help the clinician predicting the likely pathogen causing SARI [14].

Fifty-three percent of our patients had medical comorbidities, with the predominance of chronic lung diseases (43\%). The impacts of medical comorbidities on patients with SARI were addressed in previous surveillance studies $[9,13,14]$. Despite that $83 \%$ of our cohort were children less than 5 years, and patients with comorbidities were significantly older compared to those with no comorbidities, patients with and without viral detection differed significantly in the frequencies of chronic respiratory, as well as endocrine, hepatic and neuromuscular disorders.

Comparing the clinical course, complications, and outcomes between viral-infected cases and non-viral detected controls showed interesting results. Patients with identified viruses had significantly lower rates for ICU admission, hospital stay, length of mechanical ventilation, and overall mortality than those without identified viruses. However, there were no differences with regards to ARDS and mechanical ventilation.

Previous studies showed conflicting results on the impacts of viral infections on clinical outcomes in patients with SARI [9, 13, 14, 19, 22, 23]. Differences in patients' numbers, enrollment criteria, and methodologies could explain these results. Although PCR has been established as a reliable diagnostic assay with high sensitivity and specificity for respiratory viruses, particularly for RSV [24], the clinical implications of positive laboratory results are still less clear [13].

Patients with positive viral detection had better clinical outcomes than those with no viral detection, in terms of pneumonia, ICU admission, and overall mortality. Furthermore, compared to patients with no virus identified, patients with RSV-positive infection were significantly less likely to have pneumonia, to be admitted to the ICU, mechanically ventilated, and had less mortality.

Interestingly, analyses to assess associations with severe outcomes in the current study revealed that no infections were independently associated with those outcomes, even after controlling for age and associated medical comorbidities. Despite the predominance of RSV infections among SARI-positive cases (45\%), there was strong evidence that individuals with RSV and influenza were less likely to experience a severe outcome than those not infected with each of these pathogens. Furthermore, individuals with multiple infections were no more likely than those with infection with a single pathogen to experience severe outcomes.

Multivariate logistic regression analysis confirmed that individuals with positive results for rhinovirus and adults $>18$ years were more likely to experience a severe outcome than those not infected with rhinovirus and children $<18$ years, respectively. However, because of the low prevalence of rhinovirus (2\%) and adults $(8.8 \%)$ in this study, further larger studies are needed to confirm these associations.

Being the most commonly detected virus among our cohort, there was an interest to examine the RSV-positive cases. Interestingly, while patients with RSV-positive infections had significant differences with those with no respiratory viruses identified with regards to clinical signs and symptoms, comorbidities, and outcomes; they had no differences with those tested positive for other viral pathogens with regards to the same parameters.

However, individuals with RSV and associated medical comorbidities were more likely to experience severe outcomes than those with RSV and no comorbidities, after controlling for age and other risk factors.

Again, review of the literature had shown conflicting results for clinical implications of RSV infection [9, 2328]. While the relationship between RSV infection and clinical disease has been established, as infections among asymptomatic individuals are rare $[9,24-27]$, no relationship between viral load and disease severity was identified by others [23, 28, 29]. For non-influenza viruses, the clinical features are still unclear. Adenovirus infection levels in asymptomatic children and adults varied $[27,30]$, though this may be attributable to differences in sampling methodology since throat swabs may detect latent AdV DNA in tonsil tissue [27]. Studies suggest that asymptomatic infection with hMPV is rare among children [31], but results from adult populations are less conclusive, with reports of varying levels of infection among asymptomatic individuals [25, 32].

Furthermore, the clinical implications of positive laboratory results are further complicated by the presence of co-infections. Multiple viral respiratory pathogens were identified in $16.7 \%$ of our cases. Co-infection with 2 or more viral respiratory pathogens has been encountered in previous reports among pediatric populations in the Middle East [13, 18, 33, 34]. Multiple infections complicate diagnosis, as the relative clinical impact of each pathogen is unclear [13], and certain pathogens, such as adenovirus, are routinely found in the upper airways [35]. 
This study has many points of strength; it was the first surveillance that addresses the clinical impacts and epidemiological patterns of viral and atypical bacteria causing SARI in both children and adult Egyptian population, with enrolled large numbers of patients and over a relatively long period. Furthermore, analyses of homogenous populations, rather than different ethnic groups [14], give the results reliable and strong support. On the other hand, it has some limitations; more time may be needed for properly evaluating the role of atypical bacteria, and the flu vaccine was not used.

\section{Conclusions}

The current study showed that viral pathogens were encountered in one-third of hospitalized adult and pediatric Egyptian patients with SARI. Atypical bacteria had a minor role in SARI in our locality. Highest rates of viral infections were reported for RSV, PIV, and adenovirus. The presence of chronic respiratory, endocrine, hepatic and neuromuscular disorders negatively affects patients with identified viral infections. Viral infections had no negative impacts on clinical features, clinical course, and severe outcomes of SARI in our locality. Further studies are warranted.

\begin{abstract}
Abbreviations
ARDS: Acute respiratory distress syndrome; ARIs: Acute respiratory infections; CDC: Center of Disease Control; CUH: Cairo University Hospital; HBOV: Human Bocavirus; hMPV: Human metapneumovirus; Ig: Immunoglobulin; ILI: Influenza-like illness; IQR: Interquartile range; NP: Nasopharyngeal; OP: Oropharyngeal; OR: Odds Ratio; PIV: Parainfluenza virus; RSV: Respiratory syncytial virus; RT-qPCR: Quantitative real-time reverse transcription polymerase chain reaction; SARI: Severe acute respiratory infection; VTM: Viral transport medium; WHO: World Health Organization
\end{abstract}

\section{Acknowledgments}

The authors thank the nursing team at Cairo University Hospitals (CUH) and the technician teams of the laboratory departments of $\mathrm{CUH}$ and the Naval Medical Research Unit No.3 (NAMRU-3) for their contribution to this work.

\section{Funding}

This research did not receive any specific grant from funding agencies in the public, commercial, or not-for-profit sectors.

\section{Availability of data and materials}

The datasets used and/or analyzed during the current study are available from the corresponding author on reasonable request.

\begin{abstract}
Authors' contributions
All authors have provided approval of the manuscript to be published. AH, $S M, \cup A$, and El contributed to the draft of the submitted revised article, revised it critically for important content, and made substantial contributions to conception and design, as well as data acquisition, analysis, and interpretation; $\mathrm{AH}$ and $\mathrm{MR}$ contributed to the draft of the submitted article and made substantial contributions to acquisition, analysis, and interpretation of clinical data of patients admitted to the ICU. AE and $\mathrm{MH}$ contributed to the draft of the submitted article and made substantial contributions to acquisition, analysis, and interpretation of laboratory data.
\end{abstract}

\section{Ethics approval and consent to participate}

The study protocol was approved by the Institutional Review Board at the NAMRU-3, as well as the ethical committee of Cairo University Hospital $(\mathrm{CUH})$, in compliance with all applicable federal U.S. regulations governing the protection of human subjects. Informed written consent was obtained from the patients (in the case of adult patients) or patients' parent/legal guardian (in the case of pediatric patients).

\section{Consent for publication}

Not applicable.

\section{Competing interests}

The authors declare that they have no competing interests.

\section{Publisher's Note}

Springer Nature remains neutral with regard to jurisdictional claims in published maps and institutional affiliations.

\section{Author details}

'Department of Chest Diseases, Faculty of Medicine, Cairo University, Cairo, Egypt. ${ }^{2}$ Department of Chest Diseases and Tuberculosis, Faculty of Medicine, Assiut University, Assiut 71516, Egypt. ${ }^{3}$ Department of Anesthesia and Intensive Care, Faculty of Medicine, Cairo University, Cairo, Egypt. ${ }^{4}$ Department of Clinical Pathology, Faculty of Medicine, Cairo University, Cairo, Egypt. ${ }^{5}$ Department of Clinical Pathology, Faculty of Medicine, Mansura University, Mansura, Egypt.

Received: 16 November 2018 Accepted: 5 February 2019

Published online: 01 April 2019

\section{References}

1. Murray C, Lopez A, Mathers C, Stein C. The Global Burden of Disease 2000 Project: Aims, Methods, and Data Sources. Global Programme on Evidence for Health Policy. Geneva: World Health Organization; 2001. Available from; http://www.who.int/ healthinfo/paper36.pdf

2. Tregoning JS, Schwarze J. Respiratory viral infections in infants: Causes, clinical symptoms, virology, and immunology. Clin Microbiol Rev. 2010;23:74-98.

3. World Health Organization. WHO pandemic influenza draft protocol for rapid response and containment. 2006, from HTTP:// www. who.int /CSR/ disease /avian_influenza/guidelines/draftprotocol/en/index.html.

4. World Health Organization. Chapter 7: a Cough or Difficulty Breathing. In: Handbook IMCI Integrated management of childhood illness. Geneva: World Health Organization; 2005. p. 23.

5. World Health Organization. WHO global technical consultation: global standards and tools for influenza surveillance. Geneva: World Health Organization; 2011. 8-10 MARCH 2011

6. World Health Organization. WHO surveillance case definitions for ILI and SARI. 2014 https://www. who.int/influenza/ surveillance monitoring/ili_ sari_ surveillance case definition/en/.

7. Al-Toum R, Bdour S, Ayyash H. Adenovirus Infections in Jordanian Hospitalized Pediatric Patients: Prevalence and Clinical Features. J Med J. 2009:43(3):171-9.

8. El Kholy AA, Mostafa NA, El-Sherbini SA, et al. Morbidity and outcome of severe respiratory syncytial virus infection. Pediatr Int. 2013;55:283-8.

9. El Kholy AA, Mostafa NA, Ali AA, El-Sherbini SA, Ismail Rl, Magdy Rl, et al. Risk factors of prolonged hospital stay in children with viral severe acute respiratory infections. J Infect Dev Ctries. 2014;8:1285-93. https://doi.org/10.3855/idc.4682.

10. Agmy G, Mohamed S, Gad Y, Farghally E, Mohammedin H, Rashed H. Bacterial Profile, Antibiotic Sensitivity and Resistance of Lower Respiratory Tract Infections in Upper Egypt. Mediterr J Hematol Infect Dis. 2013;5(1): e2013056. https://doi.org/10.4084/MJHID.2013.056 Collection 2013.

11. Breiman RF, Cosmas L, Njenga MK, Williamson J, Mott JA, Katz MA, et al. Severe acute respiratory infection in children in a densely populated urban slum in Kenya, 2007-2011. BMC Infect Dis. 2015;15:95. https://doi.org/10. $1186 /$ s12879-015-0827-x

12. WHO information for laboratory diagnosis of pandemic (H1N1) 2009 virus in humans - revised. Available at: https:/www.who.int/csr/resources/publications/ swineflu/WHO_Diagnostic_RecommendationsH1N1_20090521.pdf?ua=1.

13. Horton KC, Dueger EL, Kandeel A, Abdallat M, El-Kholy A, Al-Awaidy S, et al. Viral etiology, seasonality and severity of hospitalized patients with severe acute respiratory infections in the Eastern Mediterranean Region, 2007-2014. PLoS One. 2017;12:e0180954

14. Wansaula Z, Olsen SJ, Casa MG, Golenko C, Erhart LM, Kammerer P, et al. Surveillance for severe acute respiratory infections in Southern Arizona, 2010-2014. Influenza Other Respir Viruses. 2016;10:161-9. https://doi.org/10 1111/irv.12360. 
15. Nguyen HKL, Nguyen SV, Nguyen AP, Hoang PMV, Le TT, Nguyen TC, et al. Surveillance of Severe Acute Respiratory Infection (SARI) for Hospitalized Patients in Northern Vietnam, 2011-2014. Jpn J Infect Dis. 2017;70:522-7.

16. Zhang C, Zhu N, Xie Z, Lu R, He B, Liu C, et al. Viral etiology and clinical profiles of children with severe acute respiratory infections in China. PLoS One. 2013;8:e72606 3135-9.

17. Nair H, Nokes DJ, Gessner BD, Dherani M, Madhi SA, Singleton RJ, et al. Global burden of acute lower respiratory infections due to the respiratory syncytial virus in young children: a systematic review and meta-analysis. Lancet. 2010;375:1545-55.

18. Shafik C, Mohareb E, Yassin AS, Amin MA, El Kholy A, El-Karaksy H, et al. Vira etiologies of lower respiratory tract infections among Egyptian children under five years of age. BMC Infect Dis. 2012;12:350-8.

19. Hess B, DeSimone J. Non-influenza Respiratory Viruses. In: Moudgal V, editor. Hospital Physician Infectious Diseases Board Review Manual. 13. Wayne: Turner White Communications Inc:; 2012. p. 5-16.

20. WHO Initiative for Vaccine Research. Respiratory syncytial virus and parainfluenza viruses. (2009) Available from: ttp://www.who.int/vaccine_ research /diseases /ari /en/index2.html.

21. Durbin A, Karron R. Progress in the Development of Respiratory Syncytia Virus and Parainfluenza Virus Vaccines. Clin Infect Dis. 2003;37:1668-77. https://doi.org/10.1086/379775

22. Meerhoff TJ, Simaku A, Ulqinaku D, Torosyan L, Gribkova N, Shimanovich V, et al. Surveillance for severe acute respiratory infections (SARI) in hospitals in the WHO European region - an exploratory analysis of risk factors for a severe outcome in influenza-positive SARI cases. BMC Infect Dis. 2015;15:1.

23. Moesker FM, van Kampen JJA, van Rossum AMC, de Hoog M, Koopmans MP, Osterhaus AD, et al. Viruses as Sole Causative Agents of Severe Acute Respiratory Tract Infections in Children. PLoS One. 2016;11(3):e0150776. https://doi.org/10.1371/journal.pone.0150776.

24. Henrickson KJ, Hall CB. Diagnostic assays for respiratory syncytial virus disease. Pediatr Infect Dis J. 2007;26(11):S36-40.

25. Falsey AR, Criddle MC, Walsh EE. Detection of respiratory syncytial virus and human metapneumovirus by reverse transcription polymerase chain reaction in adults with and without respiratory illness. J Clin Virol. 2006; 35(1):46-50

26. Hasegawa K, Jartti T, Mansbach JM, Laham FR, Jewell AM, Espinola JA, et al. Respiratory Syncytial Virus Genomic Load and Disease Severity Among Children Hospitalized With Bronchiolitis: Multicenter Cohort Studies in the United States and Finland. J Infect Dis. 2014;211:1550-9.

27. Jansen RR, Wieringa J, Koekkoek SM, Visser CE, Pajkrt D, Molenkamp R, et al, Frequent detection of respiratory viruses without symptoms: toward defining clinically relevant cutoff values. J Clin Microbiol. 2011;49:2631-6.

28. Adams $\mathrm{O}$, Weis J, Jasinska K, Vogel M, Tenenbaum T. Comparison of human metapneumovirus, respiratory syncytial virus and Rhinovirus respiratory tract infections in young children admitted to hospital. J Med Virol. 2015;87:275-80.

29. Oshansky CM, Gartland AJ, Wong SS, Jeevan T, Wang D, Roddam PL, et al. Mucosal immune responses predict clinical outcomes during influenza infection independently of age and viral load. Am J Respir Crit Care Med. 2014:189:449-62.

30. SacËkesen C, Pinar A, Sekerel B, Akyon Y, SaracËlar Y. Use of polymerase chain reaction for detection of adenovirus in children with or without wheezing. Turk J Pediatr. 2005;47(3):227 PMID: 16250306.

31. Williams JV, Harris PA, Tollefson SJ, Halburnt-Rush LL, Pingsterhaus $J \mathrm{M}$, Edwards KM, et al. Human metapneumovirus and lower respiratory tract disease in otherwise healthy infants and children. New Eng J Med. 2004, 350(5):443-50

32. Walsh EE, Peterson DR, Falsey AR. Human metapneumovirus infections in adults: another piece of the puzzle. Arch Intern Med. 2008;168(22):2489.

33. Ali S, Williams J, Chen Q, Faouri S, Shehabi A, Jundi EA, et al. Human metapneumovirus in hospitalized children in Amman, Jordan. J Med Virol. 2010;82(6):1012-6.

34. Al-Thani A, Azzam S, Abubakar H, Abdel-Hadi F, Elsheikh M, Janahi I. The role of human metapneumovirus in pediatric respiratory tract infection in Qatar. Futur Virol. 2010;5(3):355-60.

35. Feikin DR, Njenga MK, Bigogo G, Aura B, Aol G, Audi A, et al. Etiology and Incidence of Viral and Bacterial Acute Respiratory Illness among Older Children and Adults in Rural Western Kenya, 2007-2010. PLoS One. 2012;7(8): e43656.

Ready to submit your research? Choose BMC and benefit from:

- fast, convenient online submission

- thorough peer review by experienced researchers in your field

- rapid publication on acceptance

- support for research data, including large and complex data types

- gold Open Access which fosters wider collaboration and increased citations

- maximum visibility for your research: over $100 \mathrm{M}$ website views per year

At BMC, research is always in progress.

Learn more biomedcentral.com/submissions 